\title{
OTHOLOMICS
}

Revista de economía, empresa y sociedad

MISCELÁNEA. PLATAFORMAS TECNOLÓGICAS

\section{Innovación social: el porqué de un fenómeno emergente}

\section{Mariona Luis Tomás}

Innobalears. Consultora de los estudios de Economía y Empresa (UOC)

RESUMEN La innovación social es un fenómeno emergente en el que grupos de personas se ponen de acuerdo para dar respuesta a desafíos comunes. La innovación social está llegando a la sociedad a través de plataformas tecnológicas basadas en internet y las redes sociales, que permiten poner en contacto a las personas que comparten los mismos objetivos.

Los formatos de innovación social son varios, y están basados en el intercambio, el uso compartido de recursos, la transmisión de conocimiento y la confianza avalada por la reputación colectiva.

La innovación social se está consolidando porque surge a partir a cambios en los valores de los usuarios, en la demanda de servicios y en el modo como se consumen. Fenómenos como Airbnb, Uber o Goteo corresponden a la punta del iceberg de la innovación social en el mercado español, un proceso que apenas acaba de empezar.

PALABRAS CLAVE innovación social; consumo colaborativo; economía colaborativa; comunidades; inteligencia colectiva

\section{Social Innovation and how it is arriving into society}

ABSTRACT Social Innovation is an emerging phenomenon where groups of people are connected to face mutual challenges. Social innovation is arriving into society through internet based platforms and social networking that allow people with the same objectives to get in touch.

Social innovation formats are diverse, and they are based on the exchange of things and services, the shared use of resources, knowledge transmission, and reliability related to collective reputation.

Social innovation is consolidating because it is based on changes in users' values, in the demand for services, and in the way services are consumed. Phenomena like Airbnb, Uber or Goteo are the tip of the iceberg of Social Innovation in Spain, a process that is just beginning.

KEYWORDS social innovation; collaborative consumption; collaborative economy; communities; collective intelligence 


\section{Introducción}

Este artículo revisa un concepto novedoso, la innovación social, y describe la manera en la que está empezando a instalarse en la sociedad actual.

El artículo se inicia con una definición genérica del concepto y con la descripción de algunos otros conceptos asociados que deben ponerse en valor para la aplicación práctica de la innovación social.

Prosigue con una recopilación de los principales formatos emergentes sobre los cuales la sociedad se está organizando, las modalidades con las que se implanta y los elementos que la componen.

Finalmente, identifica algunos ejemplos concretos de innovación social que están emergiendo con fuerza y aporta algunas directrices sobre hacia dónde podría dirigirse la innovación social en los próximos años.

\section{Qué es la innovación social}

Los que nos dedicamos a gestión de la innovación hemos visto cómo durante los últimos veinte años la innovación ha pasado de ser una actividad exclusiva de los centros de conocimiento (universidades, centros de investigación, etc.), para abrirse también a las empresas de gran dimensión, en las que era fácil encontrar laboratorios y departamentos de I+D, y donde se hacía innovación cerrada y no compartida. Posteriormente la innovación está pasando también de las empresas (cuando vimos a pequeñas empresas que desmontaban grandes monopolios a partir de conocimiento e ideas) a las personas (que son los verdaderos promotores de la innovación) y a la sociedad en general.

Para la Unión Europea, la innovación social consiste en encontrar nuevas formas de satisfacer las necesidades sociales, que no se encuentran adecuadamente cubiertas por el mercado o el sector público. Consiste también en producir los cambios de comportamiento necesarios para resolver los grandes retos de la sociedad (Innovation Union, Comisión Europea, 2010). La forma de hacerlo es capacitando a la ciudadanía y generando nuevas relaciones sociales y nuevos modelos de colaboración.

Se trata por lo tanto de una innovación ${ }^{1}$ promovida desde las personas, y habla de cómo grupos de personas se organizan para combatir desafíos comunes.

En este contexto, han surgido algunos conceptos asociados al de innovación social que se deben considerar para entender mejor el momento histórico en el que nos encontramos respecto a la organización de las personas. Son los siguientes:

- Comunidades. Grupos de personas que se articulan en torno a un objetivo común, a un interés.

- Innovación abierta. Concepto definido por Henry Chessbrough (2006), que sostiene la teoría de que, si se comparte el conocimiento, el agente que desarrolla una tecnología es el que está en mejores condiciones de desarrollarla, y todo el entorno se ve favorecido. Este concepto se puede generalizar a entornos no tecnológicos, partiendo de la base de que una sociedad que comparte el conocimiento vive mejor.

- Economía colaborativa/consumo colaborativo². La economía colaborativa o consumo colaborativo es una rama de la innovación social (que tiene que ver con cómo se organizan las personas para combatir desafíos comunes) que se centra en el intercambio de bienes y servicios usando la tecnología como fórmula para poner en contacto a las personas y avalar su reputación. Es la actualización de la tradicional forma de compartir, dejar en préstamo, alquilar, regalar o intercambiar, que estaba muy presente en la sociedad hasta mediados de siglo pasado, a través del uso de las redes sociales y de internet, y es la forma de innovación social que está tomando más fuerza a nivel global.

1. http://es.wikipedia.org/wiki/Innovaci\%C3\%B3n_social

2. http://www.collaborativeconsumption.com/ 


\section{Para qué las comunidades hacen innovación social}

Después de un periodo de crisis global, que ha sido especialmente significativa en España y que ha sido reconocida como una crisis de valores, que ha removido los cimientos sobre los que se sustenta la sociedad, aparecen nuevos valores y nuevas formas de consumir que fomentan la inserción del individuo dentro de la sociedad y su corresponsabilidad sobre su propia vida y sobre los servicios de los que dispone. Un aspecto que está transformando las estrategias de las organizaciones empresariales.

Así, tenemos, por un lado, un cambio en los hábitos de consumo de un colectivo cada vez más amplio de personas interesadas en poder disfrutar de servicios o infraestructuras de los que su situación económica no les permite disponer en propiedad y a las que se ofrecen fórmulas alternativas.

Por otro lado, como mínimo en los países occidentales, la generalización del uso de internet permite a cualquier persona identificar a otras personas que tienen intereses, objetivos o un desafío común.

Ello da lugar a un compromiso y una tendencia cada vez mayor a compartir, a co-crear y a corresponsabilizarse de lo que ocurre a nivel social, cultural, político y económico.

Se introduce en este apartado una lista de los formatos con los que grupos de personas, las comunidades, se organizan para combatir desafíos comunes, es decir, aplicar innovación social.

\subsection{Para co-crear}

Las comunidades se unen para co-crear, para generar cosas nuevas a partir de aportaciones individuales de muchas personas. Son ejemplos de innovación para la co-creación los espacios de creación literaria compartida $\left(\right.$ Soopbook $^{3}$ ), los sistemas conjuntos de generación de documentos (Google Drive ${ }^{4}$ ), la creación de postales navideñas conjuntas (como la de la UOC 2015), etc.

\subsection{Para co-financiar}

Las comunidades también se unen para co-financiar, en el denominado crowdfunding ${ }^{5}$, financiación de iniciativas a partir de micropagos ${ }^{6}$ por parte de personas que se sienten comprometidas en una causa. Durante el año 2011 proliferó la creación de plataformas de crowdfunding ${ }^{7}$ como lugares donde unos usuarios presentan sus proyectos, que suelen tener un contenido social, cultural, emprendedor, etc., en busca de financiación. Otros usuarios de la plataforma tienen la oportunidad de contribuir a que se hagan realidad estos proyectos aportando pequeñas cantidades de dinero. Poco a poco han ido quedando las plataformas que han sido capaces de articular una comunidad mayor de usuarios. Algunos ejemplos en España son plataformas como Goteo ${ }^{8}$, Lanzanos ${ }^{9}$ o Hazloposible ${ }^{10}$.

3. http://soopbook.es/

4. https://www.google.com/intl/en/drive/

5. http://en.wikipedia.org/wiki/Crowdfunding

6. https://www.youtube.com/watch?v=jA1t5KpNb2E

7. http://www.practicalecommerce.com/articles/2853-13-Crowdfunding-Websites-to-Fund-Your-Business

8. http://goteo.org/

9. http://www.lanzanos.com/

10. http://hazloposible.org/wp/portada/ 


\subsection{Para intercambiar bienes y servicios}

Otra temática que une comunidades es la de intercambiar bienes y servicios. Así, surgen los bancos de tiempo ${ }^{11}$, en los que una hora de cada persona vale lo mismo y se intercambia de manera voluntaria tiempo entre personas. También hay intercambio de casas $^{12}$, de automóviles en distintas ciudades, etc.

\subsection{Para compartir opiniones y votar}

Las comunidades se unen para compartir opiniones y enriquecerse a partir de opiniones de otros. Este tipo de comunidades incluyen los rankings más diversos: películas, vídeos, partidos políticos, música, restaurantes, hoteles, destinos turísticos... Quizá los ejemplos donde las comunidades están más consolidadas están vinculados a la experiencia turística, con plataformas como Tripadvisor ${ }^{13}$.

\subsection{Para compartir conocimientos}

El ejemplo más significativo de la comunidad global que comparte conocimiento es sin duda Wikipedia ${ }^{14}$, en la que todo tipo de ciudadanos construye y comparte conocimientos en su idioma, donde cualquiera puede contribuir siempre que corrobore el conocimiento que comparte con referencias consolidadas. Siguiendo el concepto Wikipedia, son muchas las comunidades (especialmente en el mundo académico, científico y tecnológico) que articulan el conocimiento del que disponen con una predisposición global (con la idea de identificar y compartir todo el conocimiento existente sobre un tema determinado), en la que cada cual aporta lo que sabe para construir una teoría conjunta sobre un área determinada.

\subsection{Para compartir recursos}

Las comunidades se unen cada vez más para que todos sus miembros puedan disfrutar de servicios o cosas que no quieren o no pueden poseer. Así, hay grupos de personas que se organizan para compartir viviendas, compartir experiencias turísticas ${ }^{15}$, compartir transportes en un vehículo ${ }^{16}$, compartir barcos, etc. Es la punta del iceberg de la innovación social, la parte más visible de un movimiento que apenas está empezando y agrupa entidades como Airbnb ${ }^{17}$, Creciclando ${ }^{18}$, etc.

\section{Los elementos que componen una innovación social}

Los elementos que componen una innovación social principalmente son tres, todos imprescindibles:

- Un sentido, un objetivo en común. La innovación social se articula en torno a desafíos comunes entre personas. Estos desafíos pueden ser de lo más diverso (compartir conocimiento, obtener una vivienda vacacional, intercambiar ropa de niño, etc.), pero son el desencadenante de la innovación social.

11. https://timerepublik.com/?locale=es

12. https://www.homeexchange.com/en/?utm_source=google\&utm_medium=cpc\&utm_term=homeexchange\&utm_ campaign=English+-+Other+-+Worldwide\&mkwid=sjuP9q1y5|dc\&pcrid=48225011994\&gclid=CLPm3_XS6MMCFcPJtAod3|UAew

13. http://www.tripadvisor.es/

14. https://es.wikipedia.org/wiki/Wikipedia:Portada

15. http://es.trip4real.com/

16. http://www.blablacar.es/

17. https://www.airbnb.es/

18. http://www.creciclando.com/ 
- Una comunidad. Un grupo de personas que se compromete con el sentido o el objetivo común de la innovación social y empieza a actuar para darle respuesta. Las comunidades se suelen articular de manera informal, pero a medida que crece la innovación social se convierten en fundaciones, ONG, asociaciones, etc.

- Tecnología, en particular, internet. Se usa la tecnología para involucrar a más personas en la innovación social a partir de transmitir el sentido de las innovaciones que se llevan a cabo, para mantener la cohesión de los grupos, para contactar con otras iniciativas parecidas en otros lugares del planeta, para identificar otras iniciativas parecidas... Internet se utiliza como canal de comunicación uno a uno y como canal de comunicación colectivo a través de las redes sociales.

\section{El futuro de la innovación social}

La innovación social parte de un cambio en los valores y la visión de los usuarios, así como de la generalización del uso de internet para poner a comunidades en contacto. Aun así, como fenómeno emergente tiene algunos factores que la convierten en un elemento con cierto riesgo social: la alegalidad que rodea la innovación social (dado que no está normativizada debido a su reciente surgimiento y a la rapidez de la implantación) y la falta de antecedentes está provocando, entre las entidades y grupos de interés que se sienten afectados por esta actividad, un rechazo bajo la acusación de intrusismo, economía sumergida, baja protección de los derechos de los consumidores, falta de responsabilidad y evasión de impuestos, entre otros.

No obstante, parece que la innovación social ha venido para quedarse. Existe un consenso general entre los actores sociales y empresariales en que el movimiento podría ralentizarse desde los gobiernos, pero no se puede frenar debido a que surge de cambios en la demanda. La participación de fondos de capital riesgo en iniciativas de economía colaborativa, la comercialización de elementos que vienen de las industrias tradicionales (como por ejemplo habitaciones de hotel) a través de canales de economía colaborativa (como Airbnb) y la integración de la tecnología de la economía colaborativa con tecnologías de negocios tradicionales (como por ejemplo la plataforma de transporte Uber con la cadena hotelera Hyatt o con United Airlines) hacen pensar que la economía colaborativa se integrará como parte de las oportunidades de servicio a los usuarios.

\section{Conclusiones}

La innovación social tiene que ver con cómo se organizan las personas para combatir desafíos comunes, y se centra en compartir, co-crear, intercambiar bienes y servicios usando la tecnología como vía para poner en contacto a las personas y avalar su reputación.

La innovación social está adoptando diferentes formatos (alojamiento, transporte, intercambio de servicios, préstamos económicos, intercambio de ropa y de accesorios para bebés, grupos de consumo, financiación multitudinaria, intercambio de comida, etc.) y es un fenómeno que apenas empieza en España a desplegar su potencial, principalmente centrado en el intercambio de infraestructuras turísticas (alojamiento, transporte, restauración e intercambio de servicios) pero también en otros muchos ámbitos de tipo más orientado a la sostenibilidad social.

La innovación social ha venido para quedarse, ya que surge de cambios en la demanda, y la presión social y la rápida emergencia y consolidación de múltiples plataformas avalan su interés social.

Todo esto son parámetros que vale la pena considerar y observar con criterio desde cualquier ámbito para promover la integración adecuada del movimiento colaborativo dentro de la actividad social de cualquier entorno y entenderlo como un paso más hacia la descentralización y la independencia de las personas a través de la conciencia y la responsabilidad. 


\section{Referencias bibliográficas y webgrafía}

CHESBROUGH, H. (2006). «Open Innovation. The new imperative for creating and profiting form technology». Harvard Business Review.

http://www.internationalmonitoring.com/fileadmin/downloads/trendstudien/imo\%20Trendstudie_Howaldt_ englisch_Final\%20ds.pdf

http://link.springer.com/chapter/10.1007/978-3-642-32879-4_1

http://es.wikipedia.org/wiki/Innovaci\%C3\%B3n_social

Definición de innovación social:

https://www.youtube.com/watch?v=Sfcf-_ofKz8

Artículo teórico sobre definición y tendencias en innovación social:

http://www.internationalmonitoring.com/fileadmin/Downloads/Trendstudien/IMO\%20Trendstudie_Howaldt_ englisch_Final\%20ds.pdf

Vídeo Cápsulas de innovación, a partir del minuto 15'20", conferencia de Jaume Albaiges:

http://www.redjovesolides.org/foro/materiales/videos\#.UZyRe6LOF9w.

Definición de crowdsourcing:

http://es.wikipedia.org/wiki/crowdsourcing\#cite_ref-0

Vídeo del que le puso nombre al crowdsourcing:

http://www.youtube.com/watch?v=fO-utng3ots\&feature=player_embedded\#

Vídeo explicativo de cómo funciona el crowdfunding:

http://www.youtube.com/watch?v=ja1t5kpnb2e

Artículo crítico:

http://www.todostartups.com/bloggers/desmontando-el-crowdfunding-por-manzanerame

Mesa de crowdfunding, a partir del minuto 54'20" y presentación sobre crowdunfing:

http://www.tecnolongia.org/?p=1417\&lang=can

http://www.esade.edu/web/eng/about-esade/today/events/viewelement/287624/1/13-05-2013/

collaborative-consumption

http://www.collaborativeconsumption.com/ 


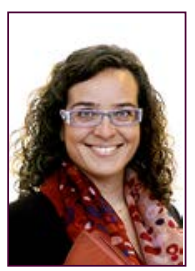

Mariona Luis Tomás

mluist@uoc.edu

Innobalears. Consultora de los estudios de Economía y Empresa (UOC))

Ingeniera industrial por la Universidad Politécnica de Cataluña, posgraduada en Gestión y planificación de proyectos por el Instituto Catalán de Tecnología, en E-learning por la Universitat Oberta de Catalunya y en un módulo de desarrollo directivo por el Instituto de Empresa.

Ha dedicado toda su vida profesional a la consultoría, en una fase inicial para empresas multinacionales como Norsistemas o Deloitte Consulting.

En 2003 volvió a Mallorca y fue directora de Innovación en el Parque Balear de Innovación Tecnológica (ParcBit), y desde el año 2005 trabaja como autónoma (bajo la marca InnoBalears) especialista en gestión de la innovación para empresas, universidades y entidades vinculadas a la Administración Pública en los ámbitos regional, nacional, europeo e internacional. Es colaboradora docente de innovación en diferentes estudios de grado y posgrado de la UOC, y desde 2009 investiga sobre innovación social y los mecanismos a partir de los cuales está llegando a la sociedad, entre los que se encuentra la economía colaborativa.

La web de InnoBalears:

http://innobalears.com/

Las entradas en el blog de InnoBalears con la etiqueta de innovación social:

http://innobalears.blogspot.com.es/search/label/innovaci\%C3\%B3n\%20social

La colaboración con temática de innovación social con la Revista Namaste:

http://www.revistanamaste.com/author/mariona/

Los textos publicados en esta revista están -si no se indica lo contrario- bajo una licencia Reconocimiento-Sin obras derivadas 3.0 España de Creative Commons. Puede copiarlos, distribuirlos y comunicarlos públicamente siempre que cite su autor y la revista y la institución que los publica (autoría, nombre de la revista, institución editora); no haga con ellos obras derivadas. La licencia completa se puede consultar en http://creativecommons.org/licenses/by-nd/3.0/es/deed.es.

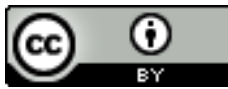

